\title{
High expression of Y-box-binding protein I correlates with poor prognosis and early recurrence in patients with small invasive lung adenocarcinoma
}

\author{
This article was published in the following Dove Press journal: \\ OncoTargets and Therapy \\ 5 May 2016 \\ Number of times this article has been viewed
}

\section{Shilei Zhao',* \\ Wei Guol,* \\ Jinxiu $\mathrm{Li}^{\prime}$ \\ Wendan $\mathrm{Yu}^{\prime}$ \\ Tao Guo' \\ Wuguo Deng' 2,3 \\ Chundong $\mathrm{Gu}^{\prime}$}

'The First Affiliated Hospital, Institute of Cancer Stem Cell, Lung Cancer Diagnosis and Treatment Center, Dalian Medical University, Dalian, ${ }^{2}$ Cancer Center, State Key Laboratory of Oncology in South China, Collaborative Innovation Center of Cancer Medicine, Sun Yat-Sen University, Guangzhou, ${ }^{3}$ State Key Laboratory of Targeted Drug for Tumors of Guangdong Province, Guangzhou Double Bioproduct Inc., Guangzhou, People's Republic of China

*These authors contributed equally to this work

Correspondence: Chundong Gu Department of Thoracic Surgery, The First Affiliated Hospital of Dalian Medical University, Zhongshan Road \#222, Dalian I I60 I I, People's Republic of China Tel +86 4I I $83635963 \times 206$ I

Fax +864 II 83622844

Email chundong2003@163.com

Wuguo Deng

Sun Yat-Sen University Cancer Center, State Key Laboratory of Oncology in South China, Collaborative Innovation Center of Cancer Medicine, Dongfeng East Road \#65I, Guangzhou 510060,

People's Republic of China

Tel +862087342282

Email dengwg@sysucc.org.cn
Background: Prognosis of small $(\leq 2 \mathrm{~cm})$ invasive lung adenocarcinoma remains poor, and identification of high-risk individuals from the patients after complete surgical resection of lung adenocarcinoma has become an urgent problem. $Y B X 1$ has been reported to be able to predict prognosis in many cancers (except lung adenocarcinoma) that are independent of TNM (tumor, nodes, metastases) staging, especially small invasive lung adenocarcinoma. Therefore, we examined the significance of YBX1 expression on prognosis and recurrence in patients with small invasive lung adenocarcinoma.

Material and methods: A total of 75 patients with small invasive lung adenocarcinoma after complete resection were enrolled from January 2008 to December 2010. Immunohistochemical staining was used to detect the expression of YBX1, and receiver operating characteristic curve analysis was performed to precisely assess the overall expression of YBX1. Meanwhile, primary lesions were identified based on the International Association for the Study of Lung Cancer, the American Thoracic Society, and the European Respiratory Society's classification of lung adenocarcinoma. The effect of different clinicopathological factors on patients' survival was examined. Furthermore, Western blot analysis was used to show the expression of YBX1 in vitro.

Results: Sensitivity and specificity of YBX1 for detecting small invasive lung adenocarcinoma from normal surrounding tissue were $66.7 \%$ and $74.7 \%$ (area under the receiver operating characteristic curve $=0.731 ; P<0.001)$, respectively. High YBX1 expression was detected in $31(41.3 \%)$ patients, and in A549, H322, Hcc827, and H1299 lung adenocarcinoma cells but not in HLF cells. In addition to sex, age, tumor size, TNM staging, pleural invasion, and lymph node metastasis, the expression of YBX1 was associated with the International Association for the Study of Lung Cancer, the American Thoracic Society, and the European Respiratory Society pathological grade risk $(P=0.026)$ and differentiation $(P=0.009)$. The patients with low YBX1 expression lived longer than those with high expression (5-year overall survival: $52.3 \%$ vs $79.0 \% ; P=0.039$ ) and showed fewer recurrences $(P=0.024)$. In multivariate analyses, high YBX1 expression (odds ratio $=2.737 ; 95 \%$ confidence interval: $1.058-7.082 ; P=0.038$ ) was shown as an independent risk factor of overall survival but not of disease-free survival (odds ratio $=1.696 ; 95 \%$ confidence interval: $0.616-4.673 ; P=0.307$ ).

Conclusion: YBX1 is an important predictor for the prognosis in patients with small invasive lung adenocarcinoma after complete resection.

Keywords: YBX1, lung adenocarcinoma, prognosis

\section{Introduction}

Lung cancer is one of the most severe malignant tumors in humans, ${ }^{1}$ while the adenocarcinoma, a leading aggressive histopathologic type of lung cancer, has become 
more and more prevalent recently. ${ }^{2}$ With the improvement of imaging and screening techniques for high-risk individuals with adenocarcinomas, ${ }^{3}$ especially those with diameter $\leq 2 \mathrm{~cm}$, it has already been detected frequently. Unfortunately, because of the malignant features of early metastasis and recurrence, patients with lung adenocarcinoma even in early stage only have the 50\%-70\% 5-year survival rate. ${ }^{4}$ Currently, the International Association for the Study of Lung Cancer, the American Thoracic Society, and the European Respiratory Society (IASLC/ATS/ERS)'s international multidisciplinary classification of lung adenocarcinoma has been widely introduced since 2011 relying on morphological characteristics and invasiveness. ${ }^{5}$ However, different gene phenotypes existing in the individuals might lead to a significant difference in their outcomes. ${ }^{6,7}$ Therefore, it is necessary to detect and identify new molecular biomarkers in order to get a more accurate prediction of prognosis and recurrence in patients with lung adenocarcinoma.

YBX1, a member of the DNA-binding protein family with an evolutionary ancient cold-shock domain, was reported to participate in the transcriptional activation of genes, the promoters of which contain an inverted CCAAT box (Y-box sequence). ${ }^{8-10}$ Upon the stimulation of outside stress, YBX1 shifted from cytoplasm to nucleus and bound to the promoter of the targeting genes, such as epidermal growth factor receptor, ${ }^{11}$ multidrug resistance $1,{ }^{12}$ and cell division cycle $6^{13}$ and, thereafter, regulated the transcription of these genes in a variety of tumor cells. Recent research has shown that the $Y B X 1$ was involved in the tumor formation, proliferation, drug resistance, and prognosis as an oncogene. ${ }^{14-16}$ Nevertheless, its expression and prognostic significances were scarcely reported in lung adenocarcinoma, especially in small $(\leq 2 \mathrm{~cm})$ invasive lung adenocarcinoma.

This study was presented to retrospectively evaluate the expression of YBX1 in patients with small invasive lung adenocarcinoma and analyze its impact on patients' prognosis and recurrence by using immunohistochemical (IHC) staining. The high YBX1 expression was found in the small invasive lung adenocarcinoma and predicted the poor prognosis.

\section{Materials and methods}

\section{Patients and follow-up}

A total of 75 consecutive patients (median age: 64 years; range from 45 years to 83 years) with small invasive lung adenocarcinoma who underwent radical surgery of the primary tumor and systematic nodal dissection at the First Affiliated Hospital of Dalian Medical University from January 2008 to
December 2010, were enrolled. The inclusion criteria of the study were based on invasive lung adenocarcinomas of the lesions $\leq 2 \mathrm{~cm}$ in diameter identified by routine histopathologic examination. The patients who received chemotherapy or radiotherapy prior to the operation were excluded. The clinical data of the enrolled patients was obtained from our institution (Lung Cancer Diagnosis and Treatment Center). The tumor stage was classified according to the seventh revision of TNM (tumor, nodes, metastases) staging of the international system for lung cancer. ${ }^{17}$

For the postoperative follow-up, we followed our previous program. ${ }^{18}$ Briefly, patients were followed every 3 months within the first year and at $\sim 6$-month intervals thereafter. During the follow-up time, physical examination, chest radiography, analysis of blood chemistry, and carcinoembryonic antigen assay were performed. If any symptom or sign of recurrence appeared in these examinations, further evaluations to detect the recurrent site were carried out. The terminal follow-up time was January 2013 (median follow-up: 32 months). The study was approved by the Medical Ethical Committees of the First Affiliated Hospital of Dalian Medical University, all patients provided written informed consent and agreed their tissue samples could be used for clinical research but not commercial use.

\section{Antibodies and other reagents}

The primary antibodies (ab12148) against YBX1 were purchased from Abcam (Cambridge, UK). The antibody against glyceraldehyde-3-phosphate dehydrogenase (GAPDH) was obtained from Proteintech Group, Inc. (Chicago, IL, USA). The Biotin-Streptavidin HRP Detection Systems was ordered from ZSGB-BIO (Beijing, People's Republic of China), and all the other chemicals were purchased from Sigma Chemicals (Perth, Australia).

\section{Histopathology evaluation}

The formalin-fixed, paraffin-embedded, $3 \mu \mathrm{m}$ section was obtained from 75 samples of primary lesions. All specimens were stained with hematoxylin and eosin for histopathologic diagnosis. Pathology assessment of each slide was independently examined by two pulmonary pathologists (Dr Li and Dr Gu) according to IASLC/ATS/ERS's international multidisciplinary classification of lung adenocarcinoma. ${ }^{5}$ Further verification was performed by the senior pathologist (Dr Sun), if there was any dispute of diagnosis between them. The invasive lung adenocarcinoma included the following: lepidic, acinar, papillary, micropapillary, solid, and mucinous predominant adenocarcinoma. 


\section{Immunohistochemistry staining}

IHC staining of YBX1 was performed in primary lesions and normal adjacent tissues according to the manufacturer's instructions of the streptavidin-peroxidase staining kit. Briefly, the sections were dewaxed, rehydrated, treated with antigen retrieval solution, serum blocked, and then incubated with polyclonal YBX1 antibody (diluted to the ratio of 1:100 in phosphate buffered saline containing $2 \%$ bovine serum albumin) overnight at $4^{\circ} \mathrm{C}$. The following day, 3,3'-diaminobenzidine (DAB) staining was performed and the result was assessed by two pathologists using a blind protocol design. Positive expression of YBX1 was indicated by pale brown cell cytoplasm, cytomembrane, and nucleus. The final overall assessed score of each specimen was calculated as intensity expression (negative, 0 points; weak, 1 points; moderate, 2 points; and strong, 3 points) multiplying with stained cell numbers (positive cells as $\leq 25 \%$ of the cells, 1 points; $26 \%-50 \%$ of the cells, 2 points; $51 \%-75 \%$ of the cells, 3 points; $>75 \%$ of the cells, 4 points).${ }^{19}$ Subsequently, the expression of YBX1 in primary lesions and normal adjacent structure was graded. To precisely assess the overall expression of YBX1 in primary lesions, receiver operating characteristic (ROC) curve analyses were carried out by two experienced pathologists based on the graded score of primary lesions and normal adjacent structure. As shown in Figure 1, when the cutoff score of YBX1 expression was 3.5 points, the maximized sensitivity and specificity of YBX1

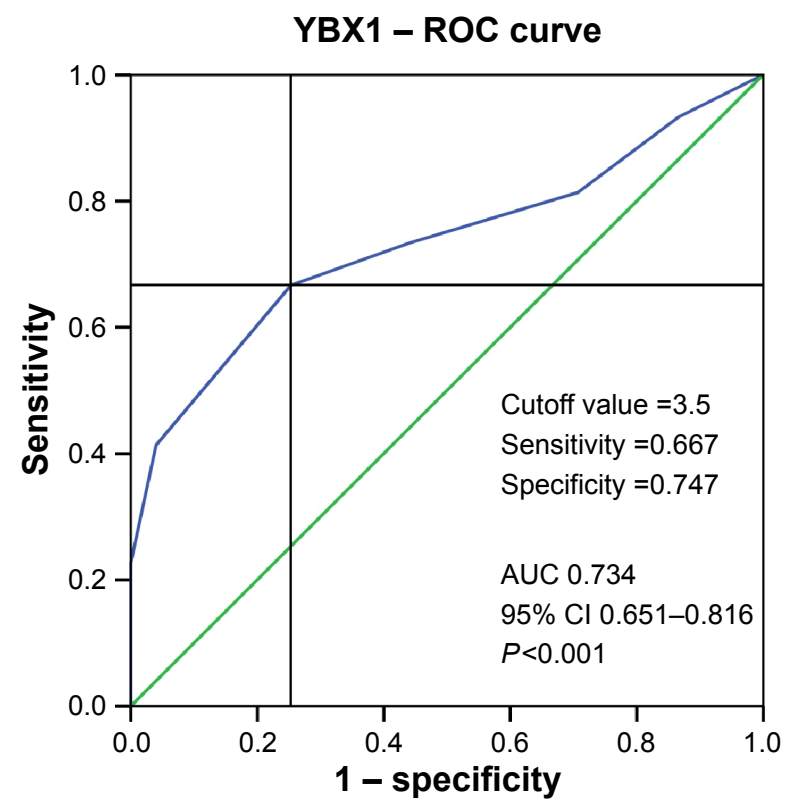

Figure I ROC curve to analyze the diacritic expression of YBXI.

Note: The AUC was 0.734 with a specificity of $74.7 \%$ and sensitivity of $66.7 \%$. Abbreviations: AUC, area under the ROC curve; $\mathrm{Cl}$, confidence interval; ROC, receiver operating characteristic. for distinguishing lung adenocarcinoma from normal human lung tissue was $66.7 \%$ and $74.7 \%$, respectively (area under the ROC curve $=0.731 ; P<0.001$ ). Therefore, YBX1 was considered to be highly expressed when the score was $>3.5$, otherwise it was considered to be less expressed.

\section{Cell lines and cell culture}

Human lung adenocarcinoma cell lines (A549, H322, Hcc827, and H1299) and normal human embryonic lung fibroblasts HLF cell line were used in this study. The A549 cells were cultured in F12K medium/Dulbecco's Modified Eagle's Medium (1:1) (HyClone; Thermo Fisher Scientific, Waltham, MA, USA) supplemented with $10 \%$ fetal bovine serum, and the other cells were grown in RPMI 1640 medium with $10 \%$ fetal bovine serum. All cells were maintained at $37^{\circ} \mathrm{C}$ in a humidified atmosphere containing $5 \% \mathrm{CO}_{2}$.

\section{Western blot analysis}

Cells grown to subconfluence were separated by trypsin digestion and collected. Total proteins were extracted using mammalian protein extraction kit (KW-BIO, Beijing, People's Republic of China) and then quantified by BCA protein assay kit (Thermo Fisher Scientific). Western blot analyses were performed according to the protocols for the routine measurement of antibodies against YBX1 (diluted 1:2,000) and GAPDH (diluted 1:5,000), respectively. Protein bands were developed with enhanced chemiluminescence system.

\section{Statistical analysis}

ROC curve analyses were performed to assess the cutoff levels of YBX1 expression, and the comparison of areas under the ROC curve was performed by $Z$-test. Statistical significance was evaluated using the chi-square test. Survival curves were plotted according to the Kaplan-Meier method, and differences between the curves were analyzed by the log-rank test. Cox proportional hazards model was applied to the multivariate survival analysis. Differences were considered to be statistically significant for $P$-values of $<0.05$. Data were analyzed using SPSS 20 software (IBM Corporation, Armonk, NY, USA).

\section{Results Clinicopathologic characteristics}

The basic clinicopathologic characteristics of 75 patients with small invasive lung adenocarcinoma are summarized in Table 1. Of 75 patients, 38 (50.7\%) were female and 37 $(49.3 \%)$ were were male; 58 (77.3\%) patients with stage Ia, seven patients with TNM stage $\mathrm{Ib}$, and ten with TNM stage 
Table I Clinicopathologic characteristics in 75 patients with completely resected small $(\leq 2 \mathrm{~cm})$ invasive lung adenocarcinoma

\begin{tabular}{|c|c|}
\hline Characteristics & Number (\%) \\
\hline Total & $75(100)$ \\
\hline \multicolumn{2}{|l|}{ Sex } \\
\hline Male & $37(49.3)$ \\
\hline Female & $38(50.7)$ \\
\hline \multicolumn{2}{|l|}{ Age } \\
\hline$\leq 64$ years & $39(52.0)$ \\
\hline$>64$ years & $36(48.0)$ \\
\hline \multicolumn{2}{|l|}{ Pathological grade risk } \\
\hline Low-grade risk (Lep) & $22(29.3)$ \\
\hline Middle-grade risk (Pap + Aci) & $40(53.3)$ \\
\hline High-grade risk $(\mathrm{Mp}+\mathrm{Sol}+\mathrm{Mc})$ & $13(17.3)$ \\
\hline \multicolumn{2}{|l|}{ Differentiation } \\
\hline Well & $39(52.0)$ \\
\hline Moderate & $3 I(4 I .3)$ \\
\hline Poor & $5(6.7)$ \\
\hline \multicolumn{2}{|l|}{ Pleural invasion } \\
\hline Absent & $67(89.3)$ \\
\hline Present & $8(10.7)$ \\
\hline \multicolumn{2}{|l|}{ Lymph node metastasis } \\
\hline Absent & $65(86.7)$ \\
\hline Present & $10(13.3)$ \\
\hline \multicolumn{2}{|l|}{ T size } \\
\hline$\leq \mathrm{I} \mathrm{cm}$ & $35(46.7)$ \\
\hline$>I \mathrm{~cm}$ and $\leq 2 \mathrm{~cm}$ & $40(53.3)$ \\
\hline \multicolumn{2}{|l|}{ TNM stage } \\
\hline la & $58(77.3)$ \\
\hline $\mathrm{lb}$ & $7(9.3)$ \\
\hline lla & $10(13.3)$ \\
\hline \multicolumn{2}{|l|}{ Surgical procedures } \\
\hline Lobectomy & $62(87.2)$ \\
\hline Wedge resection & $13(17.3)$ \\
\hline \multicolumn{2}{|l|}{ YBXI expression } \\
\hline High expression & $31(4 I .3)$ \\
\hline Low expression & $44(58.7)$ \\
\hline
\end{tabular}

Abbreviations: Aci, acinar predominant adenocarcinoma; Lep, lepidic predominant adenocarcinoma; Mc, mucinous predominant adenocarcinoma; Mp, micropapillary predominant adenocarcinoma; Pap, papillary predominant adenocarcinoma; Sol, solid predominant adenocarcinoma; TNM, tumor, node, metastases.

IIa. Ten (13.3\%) and eight (10.7\%) patients showed lymph node metastasis and pleural invasion in postoperative pathology, respectively. Lobectomy was employed on 62 patients (87.2\%), and the remaining 13 patients received partial resection. From the point of postoperative pathology, the number of patients with low, middle, and high risk of pathological grade was 22,40 , and 13 , respectively, and the number of patients with lepidic, acinar, papillary, micropapillary, solid, and mucinous predominant adenocarcinoma among the individuals was 22, 29, 11, 5, 3, and 5, respectively. During the follow-up time, $20(26.7 \%)$ of 75 patients encountered early recurrence (pulmonary metastasis, 12 cases; bone metastasis, two cases; lymphatic metastasis, 16 cases; and brain metastases one case), and $18(24.0 \%)$ patients died due to primary disease.

\section{Correlation between YBXI expression} and clinicopathologic factors

The YBX1 IHC staining is usually seen in the cytoplasm or cell membranes and few appeared in cell nucleus $(<3 \%)$. Among the 75 specimens, high YBX1 expression was detected in $31(41.3 \%)$ patients, and typical appearances of high and low YBX1 expression at different pathological grade risk are shown in Figure 2. Furthermore, as shown in Table 2, YBX1 expression was found to be positively correlated with pathological grade $(P=0.026)$ and differentiation ( $P=0.009$ ) but not with sex, age, tumor size, TNM staging, pleural invasion, and lymph node metastasis. The patients with the higher risk grade or poorer differentiation tend to have more YBX1 expression.

\section{High expression of YBXI in human lung adenocarcinoma cell lines}

We detected the expression of YBX1 in different lung adenocarcinoma cell lines (A549, H322, Hcc827, and H1299) and one normal human embryonic lung fibroblast (HLF cell line) by Western blot assay. The higher expression of YBX1 emerged in lung adenocarcinoma cell lines by comparison with HLF cells (Figure 3).

\section{Influence of risk factor on survival and recurrence}

The influence of YBX1 expression and other clinical factors on the patients' overall survival (OS) was evaluated. As shown in Figure 4 and Table 3, the Kaplan-Meier survival curves of all 75 patients demonstrated that the patients with higher pathological grade risk $(P=0.014)$, poorer differentiation $(P=0.014)$, lymph node metastasis $(P=0.037)$, elevated TNM staging $(P=0.042)$, and high YBX1 expression $(P=0.039)$ had a shorter OS. In contrast, patients with low YBX1 expression lived longer (5-year OS, $52.3 \%$ ) than those with high expression (5-year OS, 79.0\%). To our excitement, elevated TNM staging (odds ratio $[\mathrm{OR}]=3.323 ; 95 \%$ confidence interval $[\mathrm{CI}]$, $1.059-10.432 ; P=0.040$ ) and high YBX1 expression (OR $=2.737 ; 95 \%$ CI, 1.058-7.082; $P=0.038$ ) were shown as independent risk factors of OS in further multivariate analysis (Table 4). On the other hand, we found that pathological grade risk $(P=0.035)$, differentiation $(P<0.001)$, pleural invasion $(P=0.020)$, lymph node metastasis ( $P=0.036)$, TNM staging $(P=0.044)$, and YBX1 expression $(P=0.024)$ were related to the disease-free survival (DFS) in addition to the following: sex, age, surgical procedures, and tumor size (Figure 5). However, it was a pity that high expression of YBX1 (OR =1.696; 95\% CI: 0.616-4.673; 


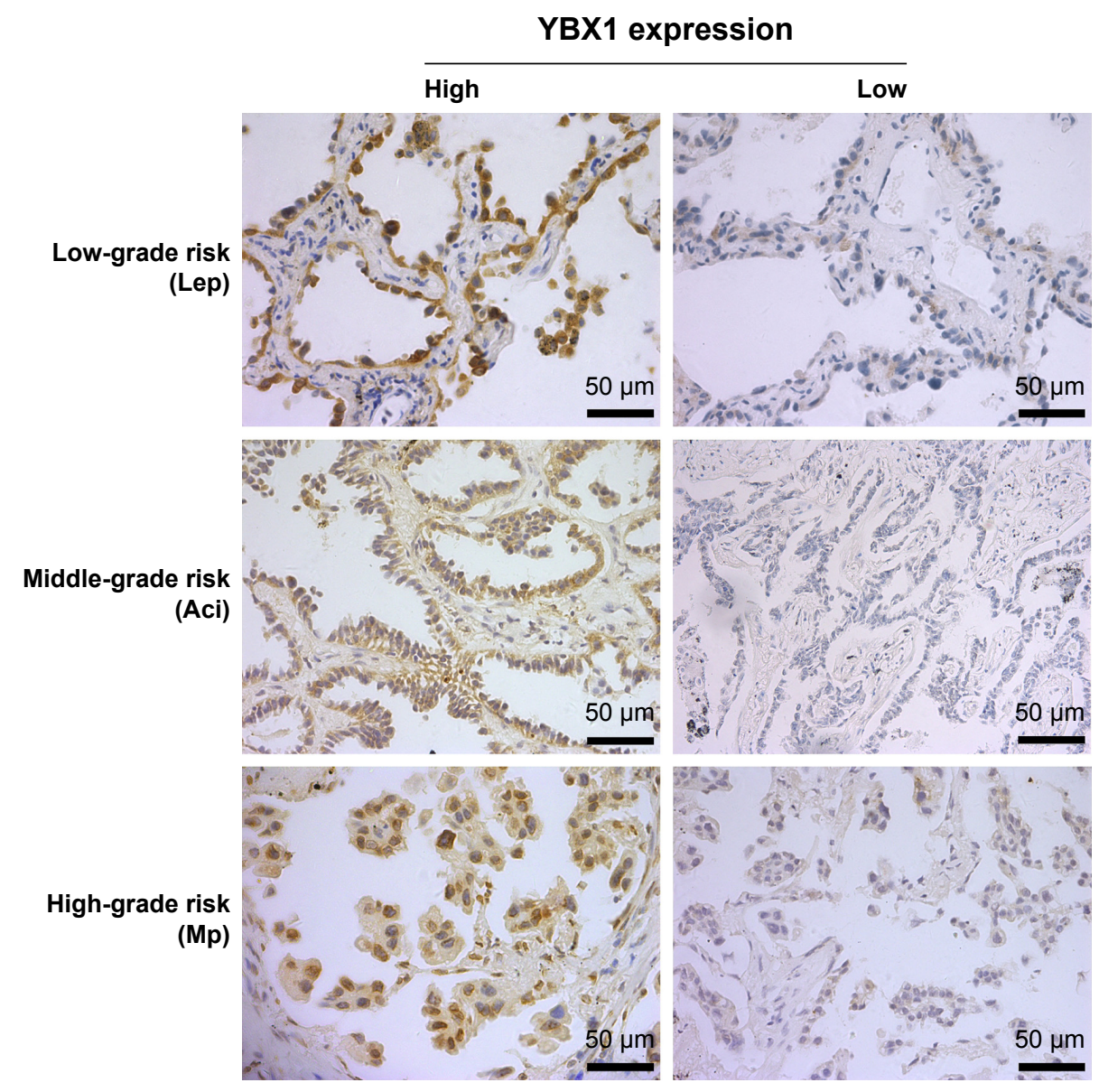

Figure 2 Expression of YBXI in different pathological grade risk group.

Notes: The grade risk groups are in accordance with IASLC/ATS/ERS's international multidisciplinary classification of lung adenocarcinoma. ${ }^{5}$ Lepidic, acinar, and micropapillary predominant growth pattern as the typical representativeness had shown high and low expression of YBXI, respectively, by immunohistochemistry (magnification $\times 400$ ). Abbreviations: IASLC/ATS/ERS, International Association for the Study of Lung Cancer, the American Thoracic Society, and the European Respiratory Society; Aci, acinar predominant adenocarcinoma; Lep, lepidic predominant adenocarcinoma; Mp, micropapillary predominant adenocarcinoma.

$P=0.307$ ) was ruled out as a confounding factor by using Cox proportional hazards model, and only the differentiation was shown as an independent risk factor of 5-year DFS in further multivariate analysis (Table 5).

\section{Discussion}

Adenocarcinoma is the main pathological type of nonsmall cell lung cancer and accounts for $>50 \%$ morbidity of lung cancer. ${ }^{2,20-22}$ In recent years, small $(\leq 2 \mathrm{~cm})$ invasive lung adenocarcinoma could be easily detected by using a wide application of low-dose spiral computed tomography screening. ${ }^{3,23}$ However, invasive adenocarcinoma shows poorer prognosis than adenocarcinoma in situ, and minimally invasive adenocarcinoma among small lung adenocarcinoma. Meanwhile, different types of invasive adenocarcinoma show different outcomes according to the IASLC/ATS/ ERS's international multidisciplinary classification of lung adenocarcinoma, ${ }^{5,24-26}$ and the different gene phenotype in individuals leads to a significant difference in outcomes.
Therefore, it is important to detect and identify new molecular biomarkers for an accurate prediction of prognosis. ${ }^{27}$ In this study, patients with small invasive lung adenocarcinoma were enrolled and the association between YBX1 expression and prognosis was retrospectively investigated to evaluate the expression of YBX1 in different human lung adenocarcinoma cell lines by comparison with normal lung fibroblast cell line.

YBX1, a multifunctional protein, was reported to be excessively activated in many malignant tumors and to be involved in cell proliferation, cell cycle, and drug resistance. ${ }^{28}$ The CSD domain of YBX1 could interact with inverted CCAAT boxes (Y-boxes) in promoter regions of some oncogenes, such as multidrug resistance 1, PCNA, and cell division cycle 6 , and thereby regulate the transcription of these genes. Shibahara et al ${ }^{29}$ reported that the positive expression of YBX1 was associated with lymph node metastasis and poor prognosis, and it may be a marker of progression in lung squamous carcinoma. To our knowledge, 
Table 2 Relations between the expression of YBXI and clinicopathologic characteristics in invasive lung adenocarcinoma

\begin{tabular}{|c|c|c|c|}
\hline \multirow[t]{2}{*}{ Characteristics } & \multicolumn{2}{|c|}{ YBXI expression } & \multirow[t]{2}{*}{$P$-value } \\
\hline & High (\%) & Low & \\
\hline Total & $3 I(4 I .3)$ & 44 & \\
\hline Sex & & & 0.740 \\
\hline Male & $16(43.2)$ & 21 & \\
\hline Female & $15(39.5)$ & 23 & \\
\hline Age & & & 0.143 \\
\hline$\leq 64$ years & $13(33.3)$ & 26 & \\
\hline$>64$ years & $18(50.0)$ & 18 & \\
\hline Pathological grade risk & & & 0.026 \\
\hline Low-grade risk (Lep) & $5(22.7)$ & 17 & \\
\hline Middle-grade risk (Pap + Aci) & $17(42.5)$ & 23 & \\
\hline High-grade risk $(\mathrm{Mp}+\mathrm{Sol}+\mathrm{Mc})$ & $9(69.2)$ & 4 & \\
\hline Differentiation & & & 0.009 \\
\hline Well & $10(25.6)$ & 29 & \\
\hline Moderate & $17(54.8)$ & 14 & \\
\hline Poor & $4(80)$ & 1 & \\
\hline Pleural invasion & & & 0.198 \\
\hline Absent & $26(38.8)$ & 41 & \\
\hline Present & $5(62.5)$ & 3 & \\
\hline Lymph node metastasis & & & 0.732 \\
\hline Absent & $26(40.0)$ & 39 & \\
\hline Present & $5(50.0)$ & 5 & \\
\hline \multicolumn{4}{|l|}{ T size } \\
\hline$\leq \mathrm{lcm}$ & $10(28.6)$ & 25 & \\
\hline$>1 \mathrm{~cm}$ and $\leq 2 \mathrm{~cm}$ & $2 \mathrm{I}(52.5)$ & 19 & \\
\hline TNM stage & & & 0.520 \\
\hline la & $22(37.9)$ & 36 & \\
\hline $\mathrm{lb}$ & $4(57.1)$ & 3 & \\
\hline Ila & $5(50.0)$ & 5 & \\
\hline
\end{tabular}

Notes: Statistical significance was evaluated using the chi-square test. Differences were considered to be statistically significant for $P$-values of $<0.05$ which are shown in bold.

Abbreviations: Aci, acinar predominant adenocarcinoma; Lep, lepidic predominant adenocarcinoma; Mc, mucinous predominant adenocarcinoma; Mp, micropapillary predominant adenocarcinoma; Pap, papillary predominant adenocarcinoma; Sol, solid predominant adenocarcinoma; TNM, tumor, node, metastases.

it might the first time the expression of YBXI in small invasive lung adenocarcinoma has been studied. In this study, the level of YBX1 was precisely assessed in primary lesions by comparison with normal adjacent tissue using ROC curve analyses. This procedure may be difficult, but it is more accurate than visual observation. Previous research showed that YBX1 was expressed in cytoplasm, but it would shift into the nuclear irradiation with ultraviolet treatment or treatment with anticancer drugs. ${ }^{30,31}$ Consistent with this, our study also detected that YBX1 protein appeared primarily in cytoplasm. We found that 31 (41.3\%) patients with small invasive lung adenocarcinoma had high YBX1 expression mainly distributed in cytoplasm, but not in nucleus $(<3 \%)$. Nevertheless, Gu et $\mathrm{al}^{32}$ reported the higher proportion of YBX1 expression

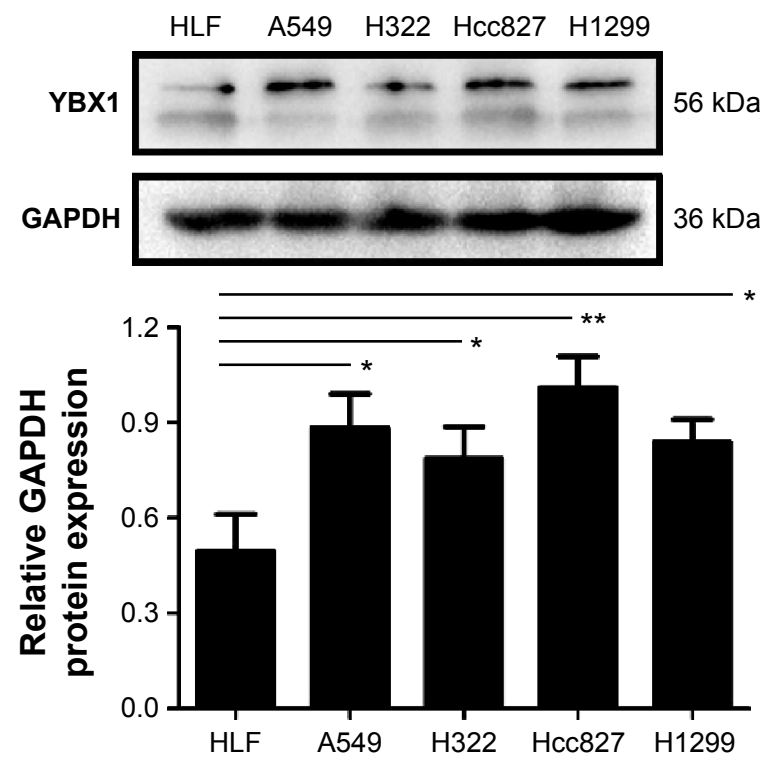

Figure 3 Analysis of total protein extracted from human lung adenocarcinoma cell lines and normal human embryonic lung fibroblasts cell line by Western blot.

Notes: Histogram was used as the quantification of YBXI expression in different cell lines. Expression of YBXI was calculated relative to the GAPDH expression. Data were repeated three times and are presented as mean $\pm S D(* P<0.05$, $* * P<0.0$ I).

Abbreviations: GADPH, glyceraldehyde-3-phosphate dehydrogenase; SD, standard deviation.

appeared in the early stage of lung cancer. The inconsistent results may be caused by the fact that our study focused on invasive adenocarcinoma and especially those with the diameter $\leq 2 \mathrm{~cm}$ in the enrolled patients. Furthermore, human lung adenocarcinoma cells such as A549, H322, Hcc827, and H1299 exhibited more expression of YBX1 than HLF cells by Western blot analysis, confirming the high expression of YBX1 in lung adenocarcinoma again.

Among all 75 patients with small invasive lung adenocarcinoma after complete surgical resection, high expression of YBX1 is significantly associated with pathological grade $(P=0.026)$ and differentiation $(P=0.009)$ but not with other clinical pathologic factors. More interestingly, the patients with the higher IASLC/ATS/ERS pathological risk grade or poorer differentiation tend to have more YBX1 expression. These findings are consistent with the report by Dahl et a ${ }^{133}$ that YBX1 expression showed a significant relationship with the differentiation in breast carcinoma $(P=0.011)$. In the high pathological risk group of patients with micropapillary and solid predominant lung adenocarcinoma, the proportion of high YBX1 expression was up to $69.2 \%$. But in the low pathological risk group of patients with lepidic predominant of adenocarcinoma, the proportion of high YBX1 expression was only $22.7 \%$. Generally, the pathological grade and differentiation were frequently used as risk factors of prognosis in different tumors. ${ }^{34,35}$ Our study found higher pathological 

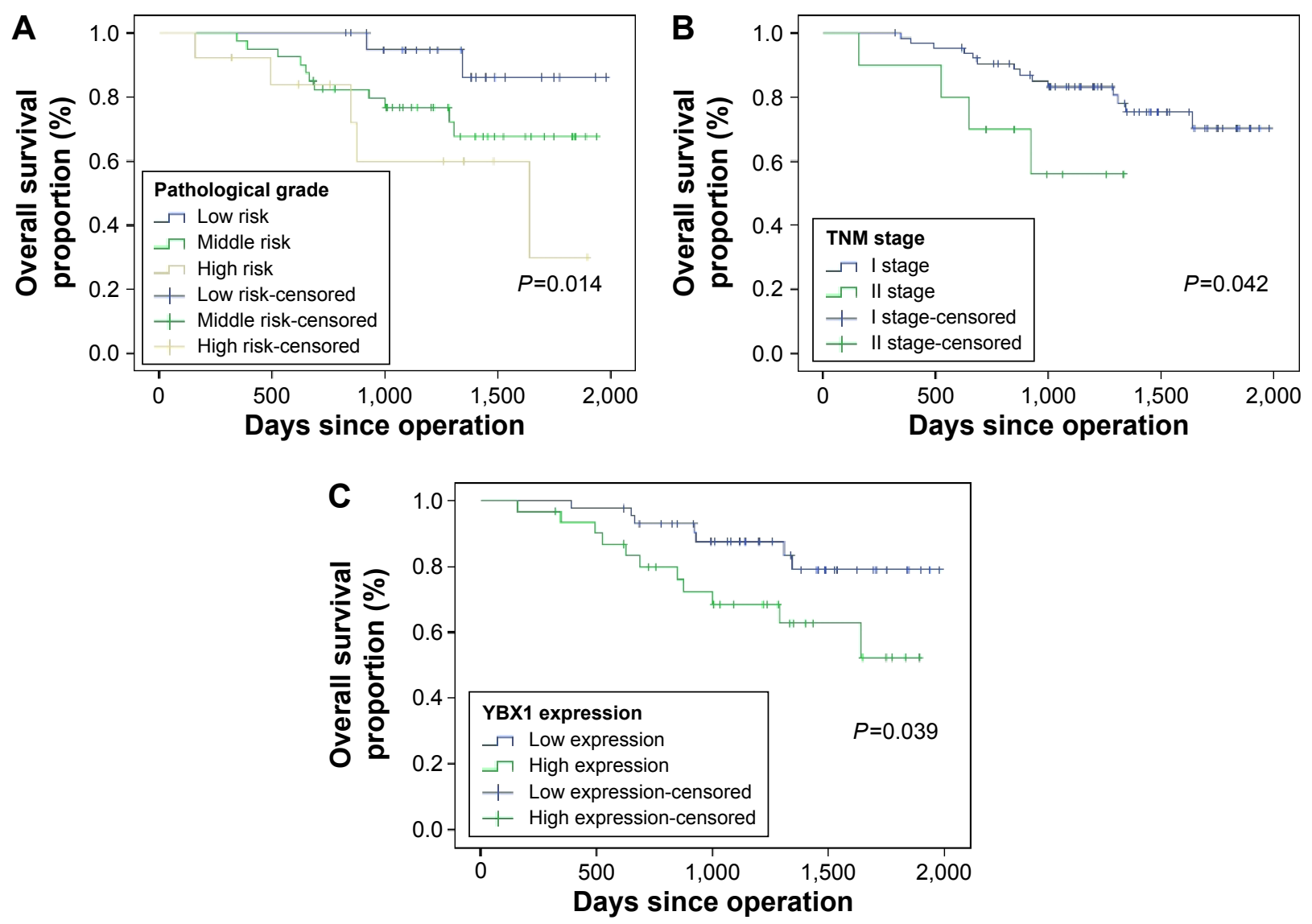

Figure 4 Kaplan-Meier survival curves for overall survival in 75 patients with small invasive lung adenocarcinoma after complete resection.

Notes: $(\mathbf{A})$ and $(\mathbf{B})$ Patients with higher pathological grade risk $(P=0.014)$ or elevated TNM staging $(P=0.042)$ had a shorter overall survival. (C) High expression of YBXI was associated with worse overall survival $(P=0.039)$ in lung adenocarcinoma.

Abbreviation: TNM, tumor, node, metastases.

Table 3 Univariate analysis of 5-year OS and disease-free survival on different clinicopathological factors using Kaplan-Meier method

\begin{tabular}{|c|c|c|c|c|}
\hline \multirow[t]{2}{*}{ Risk factor } & \multicolumn{4}{|c|}{ Kaplan-Meier method } \\
\hline & 5-OS (\%) & $\begin{array}{l}\text { Log rank } \\
\text { (P-value) }\end{array}$ & 5-FDS (\%) & $\begin{array}{l}\text { Log rank } \\
\text { (P-value) }\end{array}$ \\
\hline Sex & & 0.183 & & 0.402 \\
\hline Male & 58.0 & & 63.8 & \\
\hline Female & 76.6 & & 74.4 & \\
\hline Age & & 0.463 & & 0.747 \\
\hline$\leq 64$ years & 71.2 & & 71.1 & \\
\hline$>64$ years & 62.9 & & 67.8 & \\
\hline Pathological grade risk & & 0.014 & & 0.035 \\
\hline Low-grade risk (Lep) & 86.1 & & 82.9 & \\
\hline Middle-grade risk (Pap + Aci) & 66.7 & & 68.8 & \\
\hline High-grade risk $(M p+S o l+M c)$ & 30.0 & & 43.5 & \\
\hline Differentiation & & 0.014 & & $<0.001$ \\
\hline Well & 84.7 & & 89.0 & \\
\hline Moderate & 53.9 & & 52.9 & \\
\hline Poor & 32.3 & & 30.0 & \\
\hline Pleural invasion & & 0.080 & & 0.020 \\
\hline Absent & 69.4 & & 75.2 & \\
\hline Present & 50.0 & & 33.3 & \\
\hline
\end{tabular}


Table 3 (Continued)

\begin{tabular}{|c|c|c|c|c|}
\hline \multirow[t]{2}{*}{ Risk factor } & \multicolumn{4}{|c|}{ Kaplan-Meier method } \\
\hline & $5-\mathrm{OS}(\%)$ & $\begin{array}{l}\text { Log rank } \\
\text { (P-value) }\end{array}$ & 5-FDS (\%) & $\begin{array}{l}\text { Log rank } \\
\text { (P-value) }\end{array}$ \\
\hline Lymph node metastasis & & 0.037 & & 0.036 \\
\hline Absent & 70.3 & & 73.3 & \\
\hline Present & 58.3 & & 50.0 & \\
\hline T size & & 0.879 & & 0.207 \\
\hline$\leq \mathrm{I} \mathrm{cm}$ & 70.0 & & 76.3 & \\
\hline$>1 \mathrm{~cm}$ and $\leq 2 \mathrm{~cm}$ & 60.1 & & 64.2 & \\
\hline TNM stage & & 0.042 & & 0.044 \\
\hline 1 & 70.3 & & 73.3 & \\
\hline ॥ & 56.0 & & 50.0 & \\
\hline Surgical procedures & & 0.475 & & 0.133 \\
\hline Lobectomy & 64.0 & & 65.9 & \\
\hline Wedge resection & 83.1 & & 88.9 & \\
\hline YBXI expression & & 0.039 & & 0.024 \\
\hline High expression & 79.0 & & 79.4 & \\
\hline Low expression & 52.3 & & 54.3 & \\
\hline
\end{tabular}

Notes: Statistical significance was evaluated using the log-rank test. Differences were considered to be statistically significant for $P$-values of $<0.05$ which are shown in bold.

Abbreviations: Aci, acinar predominant adenocarcinoma; Lep, lepidic predominant adenocarcinoma; Mc, mucinous predominant adenocarcinoma; Mp, micropapillary predominant adenocarcinoma; Pap, papillary predominant adenocarcinoma; Sol, solid predominant adenocarcinoma; TNM, tumor, node, metastases; 5-FDS, 5-year disease free survival; 5-OS, 5-year overall survival.

grade risk $(P=0.014)$, poorer differentiation $(P=0.014)$, lymph node metastasis $(P=0.037)$, elevated TNM staging $(P=0.042)$, and high YBX1 expression $(P=0.039)$ affected the OS in the enrolled patients by log-rank test. In the further multivariate analysis, elevated TNM staging $(\mathrm{OR}=3.323$;

Table 4 Multivariate analysis of overall survival using Cox regression

\begin{tabular}{llll}
\hline Factors & OR & $95 \%$ Cl & $P$-value \\
\hline Pathological grade risk & & & \\
$\quad$ Low-grade risk (Lep) & 0.558 & $0.076-4.122$ & 0.568 \\
Middle-grade risk (Pap + Aci) & $\mathrm{I} .352$ & $0.236-7.754$ & 0.735 \\
$\quad$ High-grade risk (MP + Sol + Mc) & $\mathrm{I}$ & & \\
Differentiation & & & \\
$\quad$ Well & 0.190 & $0.036-1.004$ & $0.05 \mathrm{I}$ \\
$\quad$ Moderate & 0.556 & $0.192-1.61 \mathrm{I}$ & 0.279 \\
$\quad$ Poor & $\mathrm{I}$ & & \\
Lymph node metastasis & & & \\
$\quad$ Absent & 0.388 & $0.01 \mathrm{I}-13.570$ & $0.60 \mathrm{I}$ \\
$\quad$ Present & $\mathrm{I}$ & & \\
TNM stage & & & \\
II & 3.323 & $\mathrm{I} .059-10.432$ & $\mathbf{0 . 0 4 0}$ \\
I & $\mathrm{I}$ & & \\
YBXI expression & & & \\
$\quad$ High expression & 2.737 & $\mathrm{I} .058-7.082$ & $\mathbf{0 . 0 3 8}$ \\
Low expression & $\mathrm{I}$ & & \\
\hline
\end{tabular}

Notes: Statistical significance was evaluated using the Cox regression test. Differences were considered to be statistically significant for $P$-values $<0.05$ which are shown in bold.

Abbreviations: Aci, acinar predominant adenocarcinoma; $\mathrm{Cl}$, confidence interval; Lep, lepidic predominant adenocarcinoma; Mc, mucinous predominant adenocarcinoma; Mp, micropapillary predominant adenocarcinoma; OR, odds ratio; Pap, papillary predominant adenocarcinoma; Sol, solid predominant adenocarcinoma; TNM, tumor, node, metastases.
95\% CI, 1.059-10.432; $P=0.040)$ and high YBX1 expression $(\mathrm{OR}=2.737 ; 95 \% \mathrm{CI}, 1.058-7.082 ; P=0.038)$ were shown as independent risk factors of OS, while IASLC/ATS/ERS pathological grade risk exerted its prognostic values only in univariate analysis but not in multivariate analysis. By assessing the YBX1 expression, it would be possible to select high-risk patients who might benefit from early intervention at the early stage of lung adenocarcinoma. However, it was a pity to find that $\mathrm{YBX} 1$ expression $(\mathrm{OR}=1.696 ; 95 \% \mathrm{CI}$, $0.616-4.673 ; P=0.307$ ) was not associated with the patients' DFS in Cox proportional hazards model, which might be caused by the limitation of the number of patients enrolled, and further research is needed for more accurate individualized treatment.

\section{Conclusion}

In conclusion, the expression of YBX1 plays an important role in predicting prognosis, but there was no recurrence in patients with small invasive lung adenocarcinoma after complete resection. However, the limitation of this study was a small sample size, which can result in bias. Therefore, a larger sample size and randomized, and controlled clinical trials are required for further verification.

\section{Acknowledgments}

This work was supported by grants from the National Natural Science Foundation of China (81173453) and Municipal Science and Technology Program of Dalian, People's Republic 

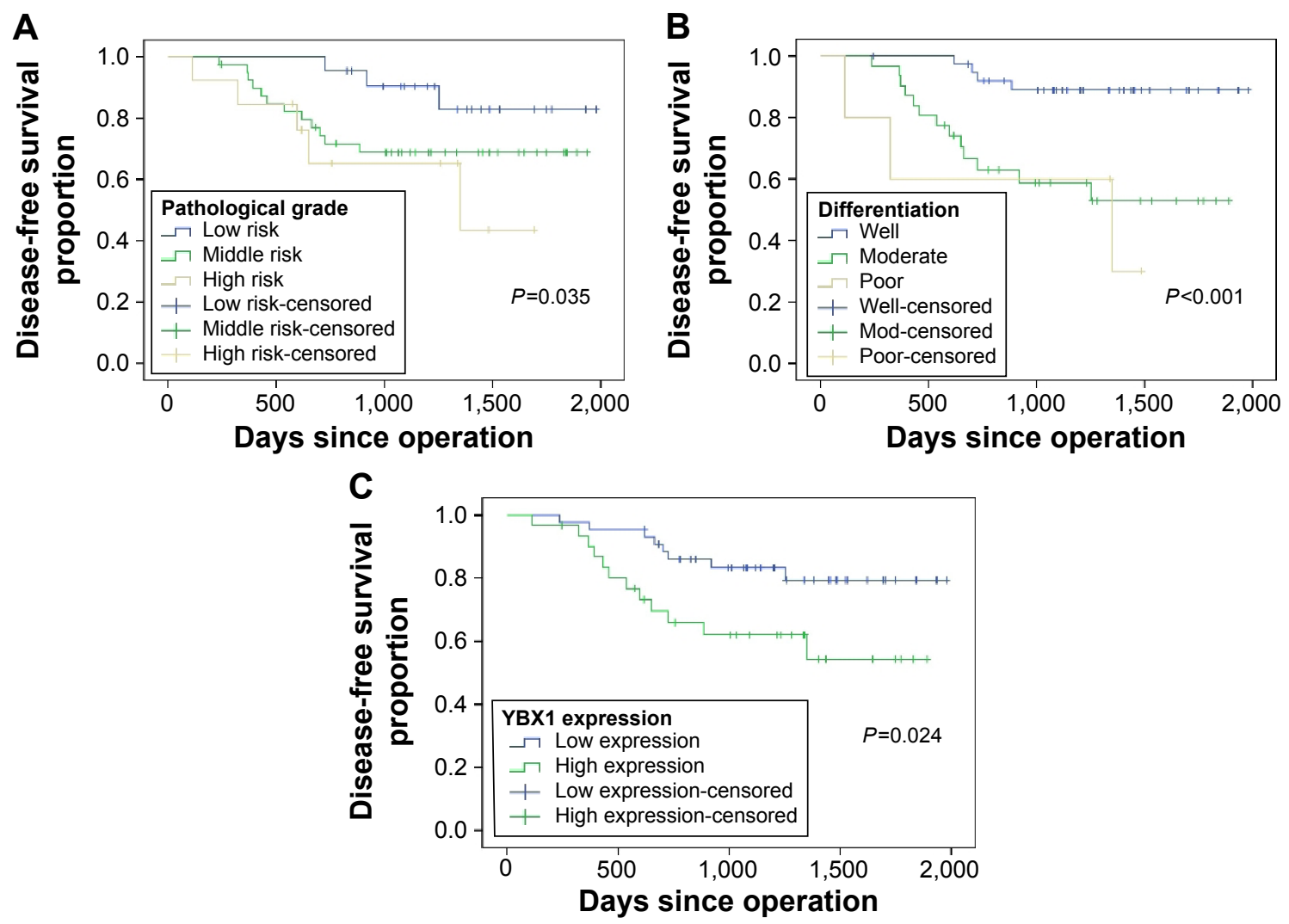

Figure 5 Kaplan-Meier survival curves for disease free survival in 75 patients with small invasive lung adenocarcinoma after complete resection.

Notes: $(\mathbf{A})$ and $(\mathbf{B})$ Patients with higher pathological grade risk $(P=0.035)$ or poorer differentiation $(P<0.00 \mathrm{I})$ had a shorter disease free survival. $(\mathbf{C})$ High expression of YBXI was associated with worse disease free survival $(P=0.024)$ compared with low expression.

Table 5 Multivariate analysis of disease-free survival using Cox regression

\begin{tabular}{|c|c|c|c|}
\hline Factors & OR & $95 \% \mathrm{Cl}$ & $P$-value \\
\hline \multicolumn{4}{|l|}{ Pathological grade risk } \\
\hline Low-grade risk (Lep) & 0.583 & $0.113-3.024$ & 0.521 \\
\hline Middle-grade risk (Pap + Aci) & 1.262 & $0.375-4.265$ & 0.706 \\
\hline High-grade risk $(\mathrm{Mp}+\mathrm{Sol}+\mathrm{Mc})$ & I & & \\
\hline \multicolumn{4}{|l|}{ Differentiation } \\
\hline Well & 0.123 & $0.028-0.553$ & 0.006 \\
\hline Moderate & 0.668 & $0.189-2.362$ & 0.531 \\
\hline Poor & I & & \\
\hline \multicolumn{4}{|l|}{ Pleural invasion } \\
\hline Absent & I & & \\
\hline Present & 2.401 & $0.808-7.128$ & 0.115 \\
\hline \multicolumn{4}{|l|}{ Lymph node metastasis } \\
\hline Absent & 0.401 & $0.016-10.129$ & 0.579 \\
\hline Present & I & & \\
\hline \multicolumn{4}{|l|}{ TNM stage } \\
\hline II & 2.383 & $0.828-6.862$ & 0.107 \\
\hline I & I & & \\
\hline \multicolumn{4}{|l|}{ YBXI expression } \\
\hline High expression & 1.696 & $0.616-4.673$ & 0.307 \\
\hline Low expression & $\mathrm{I}$ & & \\
\hline
\end{tabular}

Note: Statistical significance was evaluated using the Cox regression test. Differences were considered to be statistically significant for $P$-values $<0.05$ and marked in bold. Abbreviations: Aci, acinar predominant adenocarcinoma; $\mathrm{Cl}$, confidence interval; Lep, lepidic predominant adenocarcinoma; Mc, mucinous predominant adenocarcinoma; Mp, micropapillary predominant adenocarcinoma; OR, odds ratio; Pap, papillary predominant adenocarcinoma; Sol, solid predominant adenocarcinoma; TNM, tumor, node, metastases. of China (2012E15SF141). The authors thank Dr Zhigang Sun from the Department of Pathology, the First Affiliated Hospital of Dalian Medical University, for assistance in preparing the pathological specimens, they also thank Dr Fengzhou Li and Dr Zhipeng Tang from the Institute of Cancer Stem Cell of Dalian Medical University for data analysis.

\section{Author contributions}

All authors made substantial contributions to conception and design, acquisition of data, or analysis and interpretation of data; took part in either drafting the article or revising it critically for important intellectual content; gave final approval of the version to be published; and agree to be accountable for all aspects of the work.

\section{Disclosure}

The authors report no conflicts of interest in this work.

\section{References}

1. Siegel R, Ma J, Zou Z, Jemal A. Cancer statistics, 2014. CA Cancer J Clin. 2014;64(1):9-29.

2. Lortet-Tieulent J, Soerjomataram I, Ferlay J, Rutherford M, Weiderpass E, Bray F. International trends in lung cancer incidence by histological subtype: adenocarcinoma stabilizing in men but still increasing in women. Lung Cancer. 2014;84(1):13-22. 
3. Team NLSTR. Reduced lung-cancer mortality with low-dose computed tomographic screening. N Engl J Med. 2011;365(5):395.

4. Sawabata N. Prognosis of lung cancer patients in Japan according to data from the Japanese Joint Committee of Lung Cancer Registry. Respir Investig. 2014;52(6):317-321.

5. Travis WD, Brambilla E, Noguchi M, et al. International Association for the Study of Lung Cancer/American Thoracic Society/European Respiratory Society: international multidisciplinary classification of lung adenocarcinoma: executive summary. Proc Am Thorac Soc. 2011; 8(5):381-385.

6. Network CGAR. Comprehensive molecular profiling of lung adenocarcinoma. Nature. 2014;511(7511):543-550.

7. Wright CF, Fitzgerald TW, Jones WD, et al. Genetic diagnosis of developmental disorders in the DDD study: a scalable analysis of genome-wide research data. Lancet. 2015;385(9975):1305-1314.

8. Eliseeva I, Kim E, Guryanov S, Ovchinnikov L, Lyabin D. Y-box-binding protein 1 (YB-1) and its functions. Biochem (Mosc). 2011;76(13): $1402-1433$.

9. Annette L, Cristin G, Adele GW, Sandra ED, Antony WB. YB-1: oncoprotein, prognostic marker and therapeutic target? Biochem J. 2013; 449(1):11-23.

10. Lyabin DN, Eliseeva IA, Ovchinnikov LP. YB-1 protein: functions and regulation. Wiley Interdiscip Rev RNA. 2014;5(1):95-110.

11. Ise $\mathrm{T}$, Nagatani $\mathrm{G}$, Imamura $\mathrm{T}$, et al. Transcription factor $\mathrm{Y}$-box binding protein 1 binds preferentially to cisplatin-modified DNA and interacts with proliferating cell nuclear antigen. Cancer Res. 1999;59(2): 342-346.

12. Kuwano M, Oda $\mathrm{Y}$, Izumi $\mathrm{H}$, et al. The role of nuclear $\mathrm{Y}$-box binding protein 1 as a global marker in drug resistance. Mol Cancer Ther. 2004; 3(11):1485-1492.

13. Harada M, Kotake Y, Ohhata T, et al. YB-1 promotes transcription of cyclin D1 in human non-small-cell lung cancers. Genes Cells. 2014; 19(6):504-516.

14. Davies AH, Reipas KM, Pambid MR, et al. YB-1 transforms human mammary epithelial cells through chromatin remodeling leading to the development of basal-like breast cancer. Stem Cells. 2014;32(6): $1437-1450$.

15. Kang $\mathrm{Y}, \mathrm{Hu} \mathrm{W}$, Ivan $\mathrm{C}$, et al. Role of focal adhesion kinase in regulating YB-1-mediated paclitaxel resistance in ovarian cancer. J Natl Cancer Inst. 2013;105(19):1485-1495.

16. Liu Y, Zhao J, Zhang W, et al. IncRNA GAS5 enhances G1 cell cycle arrest via binding to YBX1 to regulate $\mathrm{p} 21$ expression in stomach cancer. Sci Rep. 2015;5:10159.

17. Sobin LH, Gospodarowicz MK, Wittekind C. TNM Classification of Malignant Tumours. New York, NY: John Wiley \& Sons; 2011.

18. Gu C-D, Osaki T, Oyama T, et al. Detection of micrometastatic tumor cells in pN0 lymph nodes of patients with completely resected nonsmall cell lung cancer: impact on recurrence and survival. Ann Surg. 2002; 235(1): 133 .

19. Zhao S, Guo T, Li J, et al. Expression and prognostic value of GalNAc-T3 in patients with completely resected small $(\leq 2 \mathrm{~cm})$ peripheral lung adenocarcinoma after IASLC/ATS/ERS classification. Onco Targets Ther. 2015;8:3143.
20. Hensing T, Chawla A, Batra R, Salgia R. A personalized treatment for lung cancer: molecular pathways, targeted therapies, and genomic characterization. Advances in Experimental Medicine \& Biology. 2014; 799(799):85-117.

21. Riessk J. Shifting paradigms in non-small cell lung cancer: an evolving therapeutic landscape. Am J Manag Care. 2013;19(19 suppl): s390-s397.

22. Zhou C. Lung cancer molecular epidemiology in China: recent trends. Transl Lung Cancer Res. 2014;3(5):270.

23. Lee HY, Han J, Lee KS, et al. Lung adenocarcinoma as a solitary pulmonary nodule: prognostic determinants of CT, PET, and histopathologic findings. Lung Cancer. 2009;66(3):379-385.

24. Kamiya K, Hayashi Y, Douguchi J, et al. Histopathological features and prognostic significance of the micropapillary pattern in lung adenocarcinoma. Mod Pathol. 2008;21(8):992-1001.

25. Yim J, Zhu L-C, Chiriboga L, Watson HN, Goldberg JD, Moreira AL. Histologic features are important prognostic indicators in early stages lung adenocarcinomas. Mod Pathol. 2007;20(2):233-241.

26. Hiroyuki S, Arafumi M, Shun-Ichi W, et al. Grade of stromal invasion in small adenocarcinoma of the lung: histopathological minimal invasion and prognosis. American Journal of Surgical Pathology. 2004; 28(2):198-206

27. Qi W, Li X, Kang J. Advances in the study of serum tumor markers of lung cancer. $J$ Cancer Res Ther. 2014;10(7):95.

28. Lage H, Surowiak P, Holm P. [YB-1 as a potential target in cancer therapy]. Pathologe. 2008;29:187-190.

29. Shibahara K, Sugio K, Osaki T, et al. Nuclear expression of the Y-box binding protein, YB-1, as a novel marker of disease progression in non-small cell lung cancer. Clin Cancer Res. 2001;7(10):3151-3155.

30. Kuwano M, Uchiumi $\mathrm{T}$, Hayakawa $\mathrm{H}$, et al. The basic and clinical implications of $\mathrm{ABC}$ transporters, Y-box-binding protein-1 (YB-1) and angiogenesis-related factors in human malignancies. Cancer Sci. 2003;94(1):9-14

31. Janz M, Harbeck N, Dettmar P, et al. Y-box factor YB-1 predicts drug resistance and patient outcome in breast cancer independent of clinically relevant tumor biologic factors HER2, uPA and PAI-1. Int $J$ Cancer. 2002;97(3):278-282.

32. Gu C, Oyama T, Osaki T, Kohno K, Yasumoto K. Expression of Y box-binding protein-1 correlates with DNA topoisomerase IIalpha and proliferating cell nuclear antigen expression in lung cancer. Anticancer Res. 2000;21(4A):2357-2362.

33. Dahl E, En-Nia A, Wiesmann F, et al. Nuclear detection of Y-box protein-1 (YB-1) closely associates with progesterone receptor negativity and is a strong adverse survival factor in human breast cancer. BMC Cancer. 2009;9(1):410.

34. Suh JH. Current readings: pathology, prognosis, and lung cancer. Semin Thorac Cardiovasc Surg. 2013;25(1):14-21.

35. Guillou L. Pleomorphic sarcomas: subclassification, myogenic differentiation and prognosis. Diagn Histopathol. 2008;14(11):527-537.
OncoTargets and Therapy

\section{Publish your work in this journal}

OncoTargets and Therapy is an international, peer-reviewed, open access journal focusing on the pathological basis of all cancers, potential targets for therapy and treatment protocols employed to improve the management of cancer patients. The journal also focuses on the impact of management programs and new therapeutic agents and protocols on

\section{Dovepress}

patient perspectives such as quality of life, adherence and satisfaction. The manuscript management system is completely online and includes a very quick and fair peer-review system, which is all easy to use. Visit http://www.dovepress.com/testimonials.php to read real quotes from published authors. 